INPLASY

PROTOCOL

To cite: Yang et al. Navel therapy for Ulcerative Colitis in adults: a systematic review and meta analysis. Inplasy protocol 2020120146. doi: 10.37766/inplasy2020.12.0146

Received: 29 December 2020

Published: 30 December 2020

Corresponding author:

Dan Yang

1042419241@qq.com

Author Affiliation:

Beijing university of Chinese medicine

Support: School project support.

Review Stage at time of this submission: Preliminary searches.

Conflicts of interest:

None.

\section{Navel therapy for Ulcerative Colitis in adults: a systematic review and meta analysis}

Yang, D1; Yan, LJ2; Mai, QL3; Guo, HL4; Hao, YF5.

Review question / Objective: The aim of this meta-analysis of randomized controlled trials is to evaluate the effect of navel therapy on symptom improvement of Ulcerative Colitis in adults.

Condition being studied: Ulcerative colitis (UC) is an idiopathic, chronic inflammatory disorder of the colonic mucosa, characterized by high rate of disease, difficult to cure and easy to repeat. It takes a toll on the quality of life of patients and is a $\mathrm{n}$ ordeal of health resources. Current medical management comprises of aminosalicylates, steroids and immunosuppressant therapies such as azathioprine, 6mercaptopurine and more recently tumor necrosis factoralpha antibody. There are some limitations in drug treatment, and non-drug alternative therapy needs to be actively explored. Navel therapy is an external treatment in traditional Chinese medicine, which is commonly used in clinical practice. Its specific operation is to prepare drugs into appropriate dosage forms (such as paste, etc.) on the umbilical cord, or to carry out some physical stimulation on the umbilical cord, such as moxibustion, cupping, massage, etc. A large number clinical trials have reported the efficacy of umbilical cord therapy in improving the symptoms of ulcerative colitis, so it is necessary to systematically analyze and integrate these trials.

INPLASY registration number: This protocol was registered with the International Platform of Registered Systematic Review and Meta-Analysis Protocols (INPLASY) on 30 December 2020 and was last updated on 30 December 2020 (registration number INPLASY2020120146).

\section{INTRODUCTION}

Review question / Objective: The aim of this meta-analysis of randomized controlled trials is to evaluate the effect of navel therapy on symptom improvement of Ulcerative Colitis in adults. 
Condition being studied: Ulcerative colitis (UC) is an idiopathic, chronic inflammatory disorder of the colonic mucosa, characterized by high rate of disease, difficult to cure and easy to repeat. It takes a toll on the quality of life of patients and is a $n$ ordeal of health resources. Current medical management comprises of aminosalicylates, steroids and immunosuppressant therapies such as azathioprine, 6-mercaptopurine and more recently tumor necrosis factor-alpha antibody. There are some limitations in drug treatment, and non-drug alternative therapy needs to be actively explored. Navel therapy is an external treatment in traditional Chinese medicine, which is commonly used in clinical practice. Its specific operation is to prepare drugs into appropriate dosage forms (such as paste, etc.) on the umbilical cord, or to carry out some physical stimulation on the umbilical cord, such as moxibustion, cupping, massage, etc. A large number clinical trials have reported the efficacy of umbilical cord therapy in improving the symptoms of ulcerative colitis, so it is necessary to systematically analyze and integrate these trials.

\section{METHODS}

Search strategy: We will search the following databases from inception until $\mathbf{3 0}$ December 2020. Relevant studies will be identified using PubMed, the Cochrane library, EmBase , Clinical Trial, Web of Science, Chinese National Knowledge Infrastructure databases (CNKI), The Chongqing Chinese Science and Technology Periodical Database (VIP), Chinese Biomedical Literature Database (CBM) and WanFang database. Trials were included with no limitations on publication type and language. Search terms include 'navel therapy' or 'shenque', AND 'Ulcerative Colitis' or 'Colitis Gravis'.

Participant or population: Adult patients $(\geq 18$ years of age) and must be diagnosed with ulcerative colitis without limitations of race, sex.
Intervention: Navel therapy, which means applying specific stimulation to the umbilical cord to achieve the therapeutic effect. The methods of stimulation include acupuncture, moxibustion, massage, drug filling, cupping, etc, or a combined stimulation method. Studies will be considered if participants in the group received navel therapy that was used alone or combined with routine pharmacotherapy (e.g. aminosalicylate ) and general adjuvant therapy or usual care.

Comparator: The comparison group will include no treatment, placebo control, usual care. If the comparison group and the Intervention group received general adjuvant therapy or usual care at the same time, the adjuvant therapy or usual care should be identical in both groups.

Study designs to be included: Randomized controlled trial(RCT).

Eligibility criteria: ALL Randomized controlled trial(RCT) about navel therapy for ulcerative colitis (UC) will be included regardless of language.The following studies: case series, quasi-RCTs Case reports, non-RCTs, cell experiments, animal experiments will be excluded. Participants:Adult patients $(\geq 18$ years of age) and must be diagnosed with ulcerative colitis without limitations of race, sex.Types of interventions: Studies will be considered if participants in the group received navel therapy that was used alone or combined with routine pharmacotherapy (e.g. aminosalicylate ) and general adjuvant therapy or usual care. the comparison group will include no treatment, placebo control, routine pharmacotherapy, usual care. If the two groups received general adjuvant therapy or usual care at the same time, the adjuvant therapy or usual care should be identical in both groups.

Information sources: We will search the following databases from inception until 1 December 2020. Relevant studies will be identified using PubMed, the Cochrane library, EmBase, Clinical Trial , Web of Science, Chinese National Knowledge 
Infrastructure databases (CNKI), The Chongqing Chinese Science and Technology Periodical Database (VIP), Chinese Biomedical Literature Database (CBM) and WanFang database.

Main outcome(s): Primary outcome : Overall symptom remission rate of Ulcerative colitis (as defined by the primary studies) ; the improvements in clinical symptoms such as abdominal pain, diarrhea, bloody purulent stool. Secondary Outcome: health-related quality of life (as defined by the primary studies) ; adverse events ;serious adverse events.

Quality assessment / Risk of bias analysis: Two researchers will independently assesses the risk of bias for all eligible studies using Cochrane Risk of Bias Tool.This tool has 7 aspects, and we will further judge each aspect as low, unclear and high risk of bias.In case of disagreement, they will consult a third researcher and reach an agreement.

Strategy of data synthesis: We will use RevMan $\mathbf{5 . 3}$ software for statistical analysis in this study. If the experiment is similar enough, we will conduct a meta-analysis on it. The mean difference (MD) or standard mean difference (SMD) were used as the effect indexes in the measurement data, and the $95 \% \mathrm{Cl}$ were given for each effect size. We will use $\times 2$ test in combination with $I^{2}$ to analyze and test the heterogeneity. If there is no statistical heterogeneity among the results, the fixed effect model is used for meta-analysis. If there is statistical heterogeneity between the results, the source of heterogeneity will be further analyzed. Significant clinical heterogeneity was treated by subgroup analysis or sensitivity analysis, or by descriptive analysis only. If the literature is sufficient, we will use funnel plots to analyze publication bias.

Subgroup analysis: We will conduct subgroup analysis based on the types of navel therapy (acupuncture, moxibustion, massage, etc.) and age group, depending on data.
Sensibility analysis: Furthermore if necessary, a sensitivity analysis will be performed.

Country(ies) involved: China.

Keywords: ulcerative colitis ; navel therapy ; effectiveness and safety ; systematic review.

Contributions of each author:

Author 1 - Dan Yang - Conceptualization; Methodology; Project administration; Writing - original draft; Writing - review \& editing.

Email: 1042419241@qq.com

Author 2 - Lijiao Yan - Conceptualization; Project administration; Validation.

Email: 731180684@qq.com

Author 3 - Qiulu Mai - Data curation;

Software; Writing - original draft.

Email: 1073764205@qq.com

Author 4 - Hailing Guo - Conceptualization; Investigation.

Email: guohailing1991@163.com

Author 5 -Yufang Hao -Conceptualization;

Supervision. 